\title{
Sistema de Controle Fuzzy Aplicado ao Ajuste de Pentano no Gás Liquefeito de Petróleo Utilizando um Sensor Virtual Baseado em Rede Neural Artificial
}

\author{
André Luiz A. Sousa* Jean Mário M. de Lima** \\ Fábio Meneghetti. U. de Araújo*** \\ * Departamento de Engenharia de Computação e Automação, \\ Universidade Federal do Rio Grande do Norte, RN, (e-mail: \\ andreluiz259@hotmail.com). \\ ** Departamento de Engenharia de Computação e Automação, \\ Universidade Federal do Rio Grande do Norte, RN, (e-mail: \\ jeanmariomlima@gmail.com) \\ *** Departamento de Engenharia de Computação e Automação, \\ Universidade Federal do Rio Grande do Norte, RN, (e-mail: \\ meneghet@dca.ufrn.br)
}

\begin{abstract}
Control techniques based on fuzzy logic have been applied as dynamic setpoint generators for controllers present in the regulatory control layer of industrial plants.This technique is called advanced control and has become widely used. This work describes the development of a fuzzy controller to perform as a dynamic setpoint generator to control the ipentane molar fraction present in the Liquefied Petroleum Gas. As a hard-to-measure variable, the amount of i-pentane will be estimated by an artificial neural network based sensor. Results have shown that the proposed system is capable of controlling the molar fraction of i-pentane. Based on a real plant located in the city of Guamare - Brazil, A simulated plant was used for testing and validation purposes.

Resumo: Técnicas de controle baseadas em lógica fuzzy vêm sendo aplicadas como geradores dinâmicos de set points para controladores presentes na camada de controle regulatório de plantas industriais. Esta técnica recebe o nome de controle avançado e tem se tornado cada vez mais utilizada. Este trabalho descreve o desenvolvimento de um controlador fuzzy para atuar como gerador dinâmico de set points para controlar a fração molar i-pentano presente no composição do Gás Liquefeito de Petróleo. Por ser uma variável de difícil medição, a quantidade de i-pentano será estimada a partir de um sensor baseado em redes neurais artificiais. Os resultados demonstram que o sistema final conseguiu ajustar a fração molar de i-pentano para todos os valores desejados. Utilizou-se uma simulação computacional, para fins de testes e validação, baseada em uma planta real que está localizada no município de Guamaré-RN.
\end{abstract}

Keywords: Fuzzy; LPG; Distillation; Artificial Neural Network; Soft Sensor; Expert Systems; Intelligent Control.

Palavras-chaves: Fuzzy; GLP; Coluna de Destilação; RNA; Sensor Virtual; Sistemas Especialistas; Controle Inteligente.

\section{INTRODUÇÃO}

A lógica fuzzy é uma das mais bem sucedidas técnicas aplicadas ao projeto controladores. A técnica surgiu a partir de estudos realizados por Zadeh (1965) e até os dias atuais é utilizada nas mais diversas aplicações no âmbito do controle de processos (Pasieka et al., 2017; Juang et al., 2017; Mendes et al., 2019). Uma dessas aplicações consiste na geração dinâmica de set points, para controladores PID's presentes na instrumentação original de plantas industriais. O objetivo é manipular determinadas variáveis do processo para obter um produto final com a qualidade desejada. Esta técnica vem sendo comumente chamada de controle avançado.

O controle avançado é aplicado em plantas de processamento de gás natural (UPGN) com a finalidade de controlar as frações molares dos elementos constituintes do produto desejado (Junior et al., 2007; Campos et al., 2009). Um produto de alto valor econômico produzido por uma UPGN é o Gás Liquefeito de Petróleo (GLP), composto idealmente de cadeias carbônicas de Propanos $\left(C_{3}\right)$ e Butanos $\left(C_{4}\right)$, porém, na prática, é inevitável a presença de contaminantes em sua composição. A fração molar ou percentual dessas substâncias consideradas contaminantes devem ser rigorosamente controladas com base em critérios 


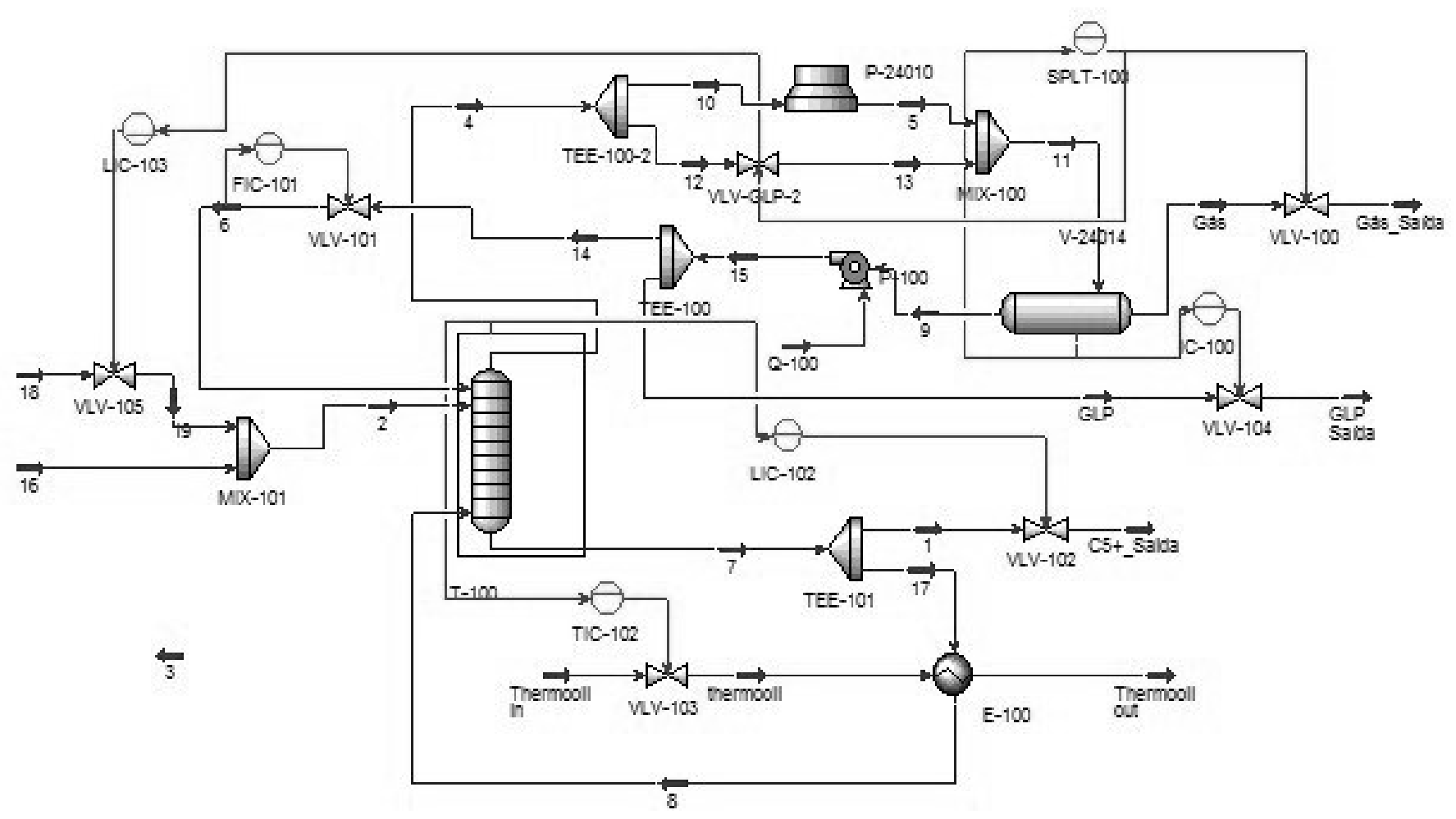

Figura 1. Coluna debutanizadora

estabelecidos pela Agência Nacional de Petróleo (ANP). Um desses elementos contaminantes presentes no GLP é o pentano, constituído pela soma de n-pentanos com ipentanos. Efetuar o controle de pentano no GLP não é uma tarefa simples devido a complexidade do processo e a dificuldade de mensurar a fração molar de pentano.

Um dos desafios encontrados para a realização do controle de variáveis de difícil medição é a falta de instrumentos de que consigam medir ou estimar essas variáveis em tempo real. Instrumentos de medição da fração molar de pentano até estão disponíveis, como os cromatógrafos a gás, entretanto apresentam longo intervalo de medição e alto custo e operação (Fortuna et al., 2005). Quando limitações técnicas e custo inviabilizam a medição das variáveis primárias, isto é, as variáveis de processo, em tempo real, sistemas de inferência - sensores virtuais apresentam-se como uma potencial solução do problema de longos intervalos para medição (Tavoosi et al., 2016). Neste trabalho, utiliza-se um sensor virtual baseado na técnica de inteligência artificial chamada de redes neurais artificiais para inferir a fração molar de pentano. Uma RNA pode mapear as relações dinâmicas não-lineares entre as variáveis primárias e secundárias de um processo com generalização adequada (Vijaya Raghavan et al., 2011). Justifica-se, assim, a implementação e utilização do sensor virtual.

Um controlador fuzzy será usado para atuar junto com o sensor virtual neural para controlar a fração molar de ipentano existente na composição do GLP que sai de uma coluna de destilação localizada na UPGN-II localizada em Guamaré-RN. O controlador será responsável por gerar set points para as malhas de controle de vazão de refluxo e temperatura de fundo da coluna, ajustando-se o perfil de temperatura da coluna, e por consequência regulando a quantidade de i-pentano. Por ser inviável a realização de testes práticos diretamente na coluna, uma simulação da mesma foi utilizada para fins de realização de testes. Optou-se por controlar somente a fração molar de ipentano pois este possui a mesma tendência de variação do n-pentano.

\section{COLUNAS DE DESTILAÇÃO}

A coluna de destilação é o aparato responsável por realizar um dos processos mais importantes na indústria de petróleo e gás, a destilação. Esse processo é responsável por separar uma mistura em seus componentes, através da adição ou subtração de calor à mistura em função das diferentes temperaturas de ebulição dos seus elementos constituintes. Um aparato de destilação é comumente composto por três elementos básicos: uma coluna, um condensador e um revedor. A coluna propriamente dita é um grande tubo cilíndrico que possui diâmetro variável de alguns centímetros a alguns metros, sendo inteiramente preenchida por pratos ou bandejas por onde passam e podem ficar acumulados líquidos e vapores. O condensador funciona como trocador de calor e é responsável por condensar o vapor que chega ao topo da coluna, resfriando-o geralmente com água ou ar frio. Assim como o condensador, o refervedor funciona como trocador de calor porém, ao invés de retirar calor da mistura presente na coluna, fornece-lhe calor para que ela chegue à torre já em estado gasoso. O refervedor situa-se na base da coluna e é responsável pela saída do produto de fundo, que é constituído pela fração mais densa da mistura presente na coluna. 


\subsection{Coluna de Destilação Debutanizadora Simulada}

A coluna de destilação utilizada neste trabalho é comumente chamada de debutanizadora e é o aparato final responsável pela produção do GLP. O produto de entrada desta coluna consiste em uma mistura formada por hidrocarbonetos de propano $\left(C_{3}\right)$, butano $\left(C_{4}\right)$, pentano e cadeias mais pesadas $\left(C_{5}+\right)$. Através do processo de destilação a coluna separa as cadeias o $C_{5}+$ dos demais compostos, fornecendo como produto de topo o GLP, formado idealmente por cadeias carbônicas de $C_{3}$ e $C_{4}$. A Figura 1 apresenta a coluna simulada no software HYSYS ${ }^{\circledR}$. Na debutanizadora, uma parte do produto depositado em seu fundo sai para a produção de $\left(C_{5}+\right)$ e outra parte passa pelo processo de aquecimento no trocador de calor E-100 retornando em seguida para a coluna. O controlador TIC102 é responsável pelo controle da vazão do óleo térmico presente no trocador de calor E-100, atuando assim no perfil de temperatura da coluna. O termo "TIC" significa "controlador indicador de temperatura", assim como o termo "FIC", que será utilizado posteriormente, significa "controlador indicador de vazão.

O produto de topo da coluna é enviado, a princípio, para um vaso acumulador de condensado podendo antes passar ou não por um condensador P-24010 (aircooler). Desse vaso, uma parte do GLP pode ser enviada à saída para ser armazenada e posteriormente comercializada, outra parte retorna a torre de destilação através de uma linha de refluxo (linha 14). O processo de refluxo, por sua vez, é controlado pelo FIC-101, controlador de vazão que atuará na válvula VLV-101, controlando a vazão de condensado que retornará a torre de destilação. Por questões de segurança uma válvula de alívio (VLV-104) pode ser acionada se houver uma pressão muito grande no vaso de condensado, neste caso, parte do GLP contido no vaso de condensado será queimado (Sousa et al., 2017).

\section{CONTROLADORES FUZZY}

Um controlador fuzzy é um sistema baseado em regras do tipo SE < premissa $>$ ENTÃO < conclusão $>$, que definem ações de controle em função das diversas faixas de valores que as variáveis de estado do problema podem assumir (Klir and Folger, 1988). Essas faixas de valores são chamadas de termos linguísticos e são quantificados através de funções de pertinência. Essas funções associam os valores discretos das variáveis de entrada a um conjunto fuzzy, convertendo os sinais de entrada em um intervalo $[0,1]$ que pode estar associado a expressões linguísticas. À partir das expressões linguísticas de entrada é gerado um modelo abstrato do sistema através de uma base de conhecimento.

A base de conhecimento fuzzy é a parte do sistema que engloba todas as regras que associarão os termos linguísticos de entrada em valores de saídas. O controlador pode ser do tipo Mamdani ou Sugeno, caso seja do tipo Mamdani, os valores de saída devem passar ainda por um processo de defuzificação, onde o valor da variável linguística de saída inferida pelas regras fuzzy será traduzido em um valor discreto. No tipo Sugeno, o valor da variável linguística de saída inferida é um valor real e são calculados diretamente através de uma média ponderada das funções Sugeno de saída, não havendo a necessidade de um processo de defu- zificação (Takagi and Sugeno, 1993). As funções Sugeno de saída podem ser valores constantes ou uma função linear das variáveis de entrada.

A lógica fuzzy tem a capacidade de incorporar a forma humana de pensar em sistemas de controle. Dessa forma, o controlador fuzzy comporta-se conforme o raciocínio que o especialista utiliza para deduzir as regras de inferência, baseadas em informações passadas já conhecidas.

\section{REDES NEURAIS ARTIFICIAIS}

Uma rede neural artificial é definida como uma estrutura distribuída, maciçamente paralela e formada em sua totalidade por unidades simples de processamento: os neurônios (Haykin, 2001). Elas tem a habilidade de aprender a partir do ambiente quando são submetidas a um processo de aprendizagem. $\mathrm{O}$ conhecimento adquirido é armazenado pela força de conexão entre os neurônios (sinapses). As principais propriedades de uma rede neural artificial são: generalização, não-linearidade intrínseca, mapeamento entrada-saída.

RNAs são ferramentas adequadas para identificação de sistemas não-lineares complexos, como colunas de destilação, pois podem associar as relações dinâmicas não-lineares entre as variáveis primárias e secundárias de um processo (Rani et al., 2013).

Como já apresentou resultados satisfatórios em sistemas de inferências (Rani et al., 2013; El-Nagar, 2018), a arquitetura de rede utilizada neste trabalho será a perceptron de múltiplas camadas (PMC). O algoritmo utilizado para treinamento é o de retropropagação de erros (backpropagation), comumente utilizado no aprendizado supervisionado.

\subsection{Identificação Utilizando Modelos Neurais}

As estruturas de modelagem baseadas em RNAs com propriedades para identificação de sistemas não-lineares são generalizações das que modelam sistemas lineares. Por exemplo, a estrutura de modelagem linear: ARX (AutoRegressive eXogenous Input). Modelos como esse apresentam um vetor que guarda valores atrasados das variáveis em uso para inferência da saída. A esse vetor dá-se o nome de vetor de regressão e, é esse vetor que caracteriza a estrutura de modelo neural de identificação.

No ARX, o vetor de regressão, não só armazena valores passados das variáveis de entrada usadas para inferir as saídas, mas também armazena os valores atrasados da própria saída. Esse modelo é estável pois apresenta associações puramente algébricas entre as variáveis de entrada e estimadas (Norgaard et al., 2001). Devido a isso, a estrutura de modelagem neural para compor o sensor virtual é o modelo chamado NNARX (Neural Network $A R X)$.

\section{SENSOR VIRTUAL PROPOSTO}

\subsection{Entradas e Saídas}

A fração molar de pentano $\left(C_{5}\right)$, composto de interesse deste trabalho, é variável primária $(V P)$ para ser inferida. Logo $C_{5}$ é a saída da rede. 
O sistema simulado almeja assegurar características reais da planta UPGN-II GMR. Portanto, apenas considera-se valores de variáveis secundárias que podem ser obtidos através de sensores físicos presentes na planta real. Para a inferência, escolheu-se a temperatura do estágio 16 $\left(V S_{1}\right)$ e vazão de refluxo $\left(V S_{2}\right)$ da coluna debutanizadora baseando-se na dinâmica que as variáveis secundárias determinam sobre as primárias. As variáveis secundárias 1 e 2 estão associadas aos controladores PID TIC-102 e LIC-101 da planta.

\subsection{Estrutura do modelo}

Durante o treinamento, utiliza-se a estrutura NNARX (Neural Network $A R X$ ), com a rede sendo realimentada por valores de saída do sistema. Nas validações, testes e aplicação junto ao controlador fuzzy, a realimentação da rede neural do sistema é dada pelas próprias estimativas da rede das frações molares do $C_{5}$.

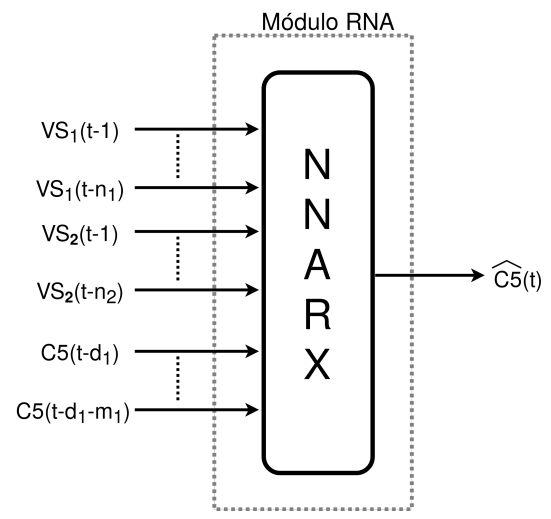

Figura 2. Diagrama esquemática da estrutura de modelagem NNARX proposta.

A Figura 1, é o esquema do modelo RNA para treinamento. No lado esquerdo da estrutura, $V S_{1}$ e $V S_{2}$ representam o vetor de regressão das duas variáveis secundárias adotadas. C5 representa o vetor de regressão formado pela variável primária na saída. Os valores descrito até aqui são coletados do próprio sistema simulado. Já $\widehat{C 5}$ é a variável primária inferida no treinamento. $\mathrm{O}$ termo $n$ representa a ordem do vetor de regressão. Já $m$ é a ordem de regressão do vetor formado por C5 que realimenta o modelo. Por fim, $d$ é o atraso de cada uma das realimentações. Para utilização do modelo após treinado, a realimentação é feita com valores que estão sendo inferidos pela própria rede rede.

\subsection{Módulo de Correção}

As estimativas geradas pelo sensor podem divergir acentuadamente dos valores esperados. A cada realimentação com as próprias estimativas do sensor, há um erro que é acumulado e propagado. Devido a isso, propõe-se um módulo de correção. Utilizando-se medições de pentano realizadas pelos cromatógrafos de linha do processo, quando disponíveis ou em determinado intervalo de tempo para realimentar o sistema.

Com a descrição individual dos módulos,apresenta-se os sistema por completo.

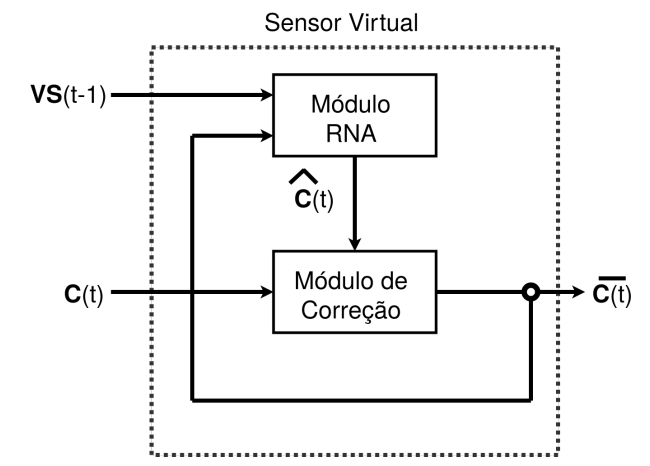

Figura 3. Esquemático do sensor virtual proposto.

VS, C, $\widehat{C}, \bar{C}$ são vetores e correspondem, respectivamente, às variáveis de secundárias dos controladores da UPGN, às medições de frações molares de $C_{5}$ fornecidas pelos cromatógrafos, às frações molares estimadas pela rede neural e, por fim, as variáveis primárias corrigidas ou não pelo módulo de correção.

\section{SISTEMA DE CONTROLE PROPOSTO}

Um controlador fuzzy do tipo Sugeno foi desenvolvido para atuar junto com o sistema de inferência neural proposto. O Sistema recebe como entrada o erro e a variação do erro relacionados a quantidade de i-pentano na composição do GLP. A quantidade de i-pentano é determinada pelo sensor virtual neural. A Figura 4 apresenta um esquema do sistema fuzzy junto com o sistema de inferência atuando sobre a coluna. A rede recebe como entrada a temperatura de fundo e a vazão de refluxo da coluna debutanizadora, essas variáveis são de fácil medição e podem ser fornecidas por sensores comuns. Em seguida, é passado para o controlador fuzzy a quantidade estimada de i-pentano presente no GLP naquele determinado instante de medição. O controlador, por sua vez, fornecerá os set points para os controladores PID's da coluna à fim de se atingir a fração molar de ipentano desejada.

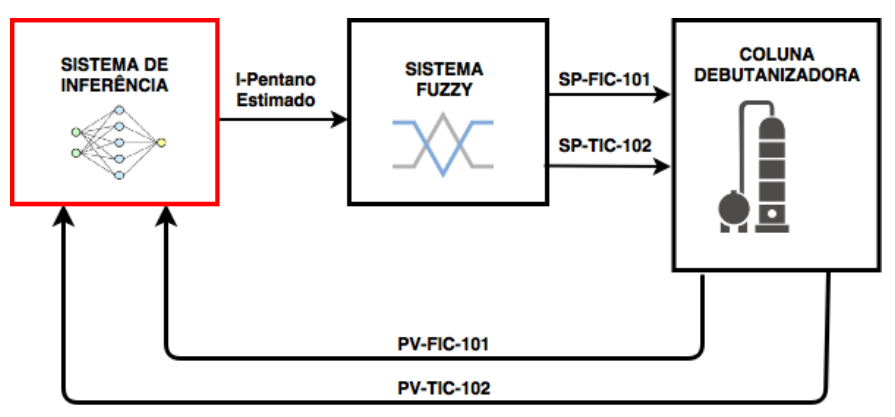

Figura 4. Esquema de controle proposto

O controlador foi projetado no software MATLAB ${ }^{\circledR}$, com período de amostragem de 30 segundos. A comunicação entre os controladores fuzzy e as colunas, simuladas no HYSYS ${ }^{\circledR}$, foi realizada através de acesso direto as variáveis do programa. A estrutura do $\mathrm{HYSYS}^{\circledR}$ fornece todo o suporte necessário para a importação e exportação de variáveis através de variáveis de ambiente do Windows ${ }^{\circledR}$. As funções de pertinências que quantificam as variáveis de entrada do sistema são do tipo bell-shaped, definidas pela seguinte equação: 


$$
f(x ; a, b, c)=\frac{1}{1+\left|\frac{x-c}{a}\right|^{2 b}}
$$

Utilizou-se cinco funções de pertinências na definição das variáveis de entrada, sendo elas: Positivo Grande (PG), Positivo (P), Zero (Z), Negativo (N) e Negativo Grande $(\mathrm{NG})$. As Figuras 5 e 6 apresentam as funções de pertinência utilizadas para as variáveis de entrada erro e variação do erro, respetivamente.

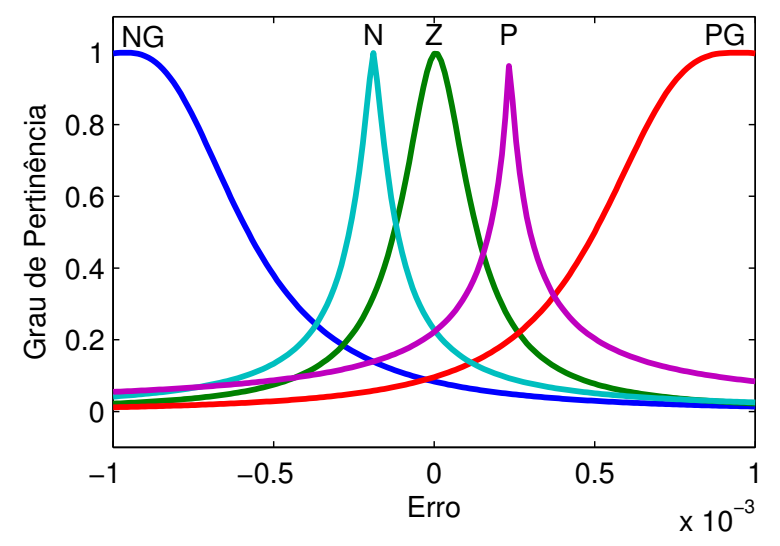

Figura 5. Funções de pertinência para a variável Erro relacionada ao i-pentano

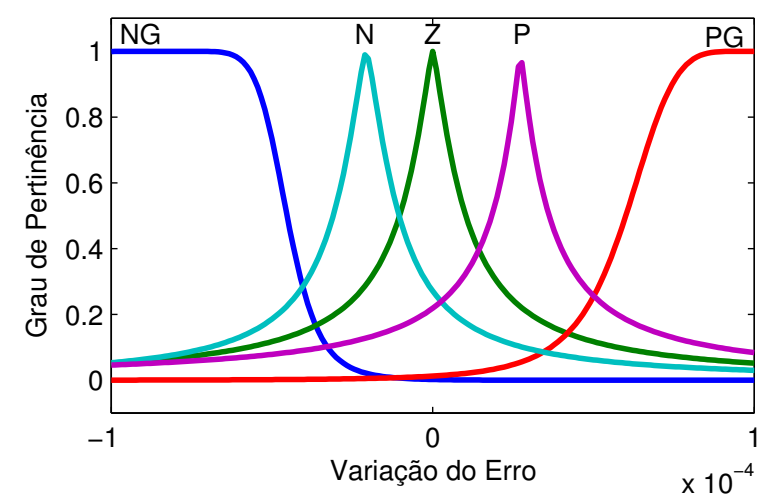

Figura 6. Funções de pertinência para a variável variação do erro relacionada ao i-pentano

As especificações dos Parâmetros a, b e c de cada uma das funções apresentadas podem ser consultados nas las 1 e 2. Esses parâmetros foram definidos com base no universo de discurso definido para o erro, e partir deste universo, definiu-se de forma empírica os valores adequados para cada uma das funções.

Tabela 1. Valores dos parâmetros das funções de pertinência para a variável erro

\begin{tabular}{cccc} 
& $\mathrm{a}$ & $\mathrm{b}$ & $\mathrm{c}$ \\
\hline $\mathrm{NG}$ & $3,84 \times 10^{-4}$ & 1,30 & $-9,64 \times 10^{-4}$ \\
$\mathrm{~N}$ & $7,51 \times 10^{-5}$ & 0,658 & $-1,89 \times 10^{-4}$ \\
$\mathrm{Z}$ & $-1,29 \times 10^{-4}$ & 0,924 & $5,29 \times 10^{-6}$ \\
$\mathrm{P}$ & $6,38 \times 10^{-5}$ & 0,48 & $2,35 \times 10^{-4}$ \\
$\mathrm{PG}$ & $4,46 \times 10^{-4}$ & 1,49 & $9,46 \times 10^{-4}$ \\
\hline
\end{tabular}

Tabela 2. Valores dos parâmetros das funções de pertinência para a variável variação do erro

\begin{tabular}{cccc} 
& $\mathrm{a}$ & $\mathrm{b}$ & $\mathrm{c}$ \\
\hline $\mathrm{NG}$ & $3,89 \times 10^{-5}$ & 3,91 & $-8,82 \times 10^{-5}$ \\
$\mathrm{~N}$ & $-1,02 \times 10^{-5}$ & 0,70 & $-2,07 \times 10^{-5}$ \\
$\mathrm{Z}$ & $-1,03 \times 10^{-5}$ & 0.639 & 0,00 \\
$\mathrm{P}$ & $8,94 \times 10^{-6}$ & 0.57 & $2,73 \times 10^{-5}$ \\
$\mathrm{PG}$ & $3,78 \times 10^{-5}$ & 2.28 & $9,78 \times 10^{-5}$ \\
\hline
\end{tabular}

Definidas quantitativamente as variáveis de entrada do sistema pode-se construir uma base de regras e inferência fuzzy que será responsável por associar determinada entrada a uma função Sugeno de saída associada. O sistema de inferência foi construído a partir de conhecimento de estudos realizados sobre a coluna. Foram feitos diversos testes para observar a resposta do sistema, formando assim uma base de conhecimento especialista a qual foi fornecida para a base de regras fuzzy. A base de regras para o controlador proposto nesta seção é mostrada nas las 3 e 4. A primeira apresenta as variáveis de saída para o controlador FIC-101, a segunda, para o controlador TIC102. O sistema possui uma base de regras de inferência com 25 regras lógicas do tipo:

Se erro é $A_{1}$ e variação de erro é $A_{2}$ então $u_{1}$ é $B_{1}$ e $u_{2}$ é $B_{2}$.

onde $A_{1}$ e $A_{2}$ são as funções de pertinência de entrada definidas como: Negativo Grande (NG), Negativo (N), Zero (Z), Positivo (P) e Positivo Grande (PG). As Variáveis $u_{1}$ e $u_{2}$ são os set points para os controladores FIC-101 e TIC-102 respectivamente, que são dados pelas funções Sugeno de saída $B_{1} B_{2}$, definidas como: Diminuir Muito (DM), Diminuir (D), Diminuir Pouco (DP), Manter (M), Aumentar Pouco (AP), Aumentar (A) e Aumentar Muito (AM).

Tabela 3. Base de regras (FIC-101).

\begin{tabular}{ccccccc}
\multicolumn{7}{c}{ Variação do erro } \\
\hline \multirow{4}{*}{ Erro } & NG & NG & N & Z & P & PG \\
& N & AM & AP & AM & AP & DP \\
& Z & A & AP & M & DP & A \\
& P & DM & DP & D & DM & D \\
& PG & A & AP & DM & DP & DM \\
\hline
\end{tabular}

Tabela 4. Base de regras (TIC-102).

\begin{tabular}{ccccccc}
\multicolumn{7}{c}{ Variação do erro } \\
\hline \multirow{4}{*}{ Erro } & NG & NG & D & Z & P & PG \\
& N & D & DP & D & DP & DP \\
& Z & D & M & M & AP & A \\
& P & DM & AP & M & A & A \\
& PG & DM & D & AP & A & AM \\
\hline
\end{tabular}

Os valores dos setpoints fornecidos pelo sistema fuzzy aos controladores são funções Sugeno constantes e serão somados ao valor anteriormente presente nos controladores FIC101 e TIC-102. A tabela 5 explicita os valores numéricos para cada uma das funções de saída do controlador. 
Tabela 5. Funções Sugeno utilizadas na geração do sinal de saída do fuzzy.

\begin{tabular}{cccccccc} 
& DM & D & DP & Manter & AP & A & AM \\
\hline FIC-101 & $-0,05$ & $-0,035$ & $-0,015$ & 0 & 0,025 & 0,035 & 0,05 \\
TIC-102 & $-0,03$ & $-0,02$ & $-0,01$ & 0 & 0,01 & 0,02 & 0,03 \\
\hline
\end{tabular}

\section{RESULTADOS}

\subsection{Treinamento e Validação das RNAs de inferência}

Para treinamento e validação da rede neural que compõe o sensor virtual, utilizou-se o toolbox nntool do software MATLAB ${ }^{\circledR}$. Implementou-se quatro redes neurais distintas, com uma camada oculta cada, com diversos valores de neurônios na camada oculta e sendo cada neurônio ativado por uma função sigmóide. A ordem de cada rede foi fixada em 2, tanto para entradas quanto para saídas, com a finalidade de diminuir a complexidade do modelo. Para cada rede realizou-se oito treinamentos com 500 épocas cada. Logo após cada treinamento, duas validações a partir de dois conjuntos de dados distintos com objetivo de atestar a capacidade de generalização das redes. Assim, obteve-se um total de 32 redes e 64 validações.

\subsection{Análise das Estruturas Neurais}

Para analisar a capacidade de generalização de cada rede, utilizou-se o erro médio quadrático (EMQ) como critério. O EMQ foi calculado para cada uma das 64 validações. Expõe-se os melhores resultados por estrutura, de acordo com seus respectivo número de neurônio na camada oculta. Assim, é possível escolher a melhor estrutura para compor o sensor virtual que trabalhará junto ao controlador fuzzy.

Tabela 6. Estatísticas de validações da inferência de pentano.

\begin{tabular}{cccc} 
Ordem & $N_{N E}$ & EMQ pentano & Erro (\%) \\
\hline 2 & 8 & $1.3991 \times 10^{-6}$ & 4.2612 \\
2 & 16 & $2.9241 \times 10^{-6}$ & 6.7503 \\
2 & 20 & $5.1732 \times 10^{-6}$ & 7.4512 \\
2 & 25 & $9.0721 \times 10^{-6}$ & 8.01782 \\
\hline
\end{tabular}

Exibe-se as médias dos EMQs calculados a partir das validações das melhores redes. $N_{N E}$ representa o número de neurônios da camada oculta. A rede composta por 8 neurônios na camada oculta teve melhor desempenho obtendo menor EMQ. Logo, a estrutura de 8 neurônios é escolhida para compor o sensor virtual que operará gerando estimativas de $C_{5}$ para o controlador fuzzy.

\subsection{Controle de I-Pentano utilizando sensor neural}

A partir das estimativas geradas pela rede neural descrita na seção 7.1 efetuou-se o controle de i-pentano através de um controlador fuzzy. A figura 7 apresenta o controle de i-pentano feito para referências escolhidas de forma aleatória. A primeira referência foi para 0.0209 a segunda 0.0205 e, por fim, 0.0214 .

Para a primeira e segunda referência, observou-se o valor de i-pentano em um intervalo de 400 períodos de amostragem, totalizando 12000 segundos. Para o ultimo degrau mediu-se por 800 períodos de amostragem, totalizando

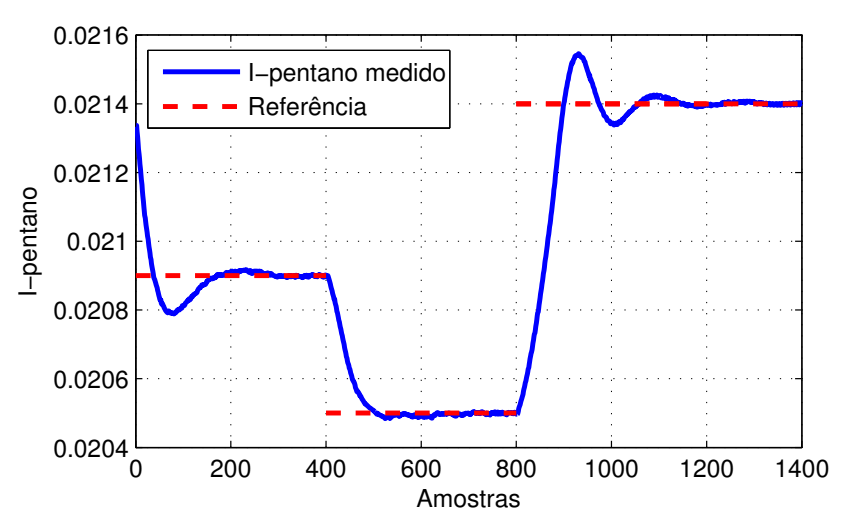

Figura 7. Fração molar de I-Pentano ajustada a partir dos controladores FIC-101 e TIC-102

24000 segundos. O tempo de acomodação para o caso mais crítico foi de aproximadamente 9000 segundos.

O set points gerados para os controlados FIC-101 e TIC102 são mostrados nas Figuras 8 e 9.

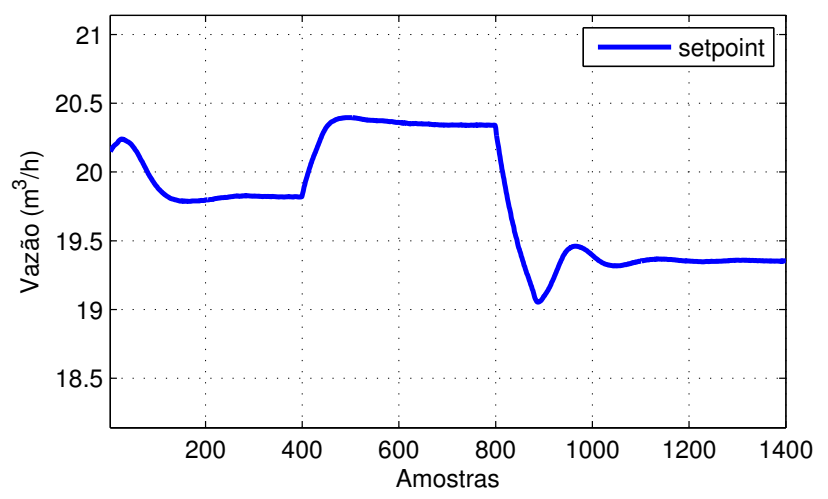

Figura 8. Set Point gerado pelo sistema fuzzy para o controlador FIC-101

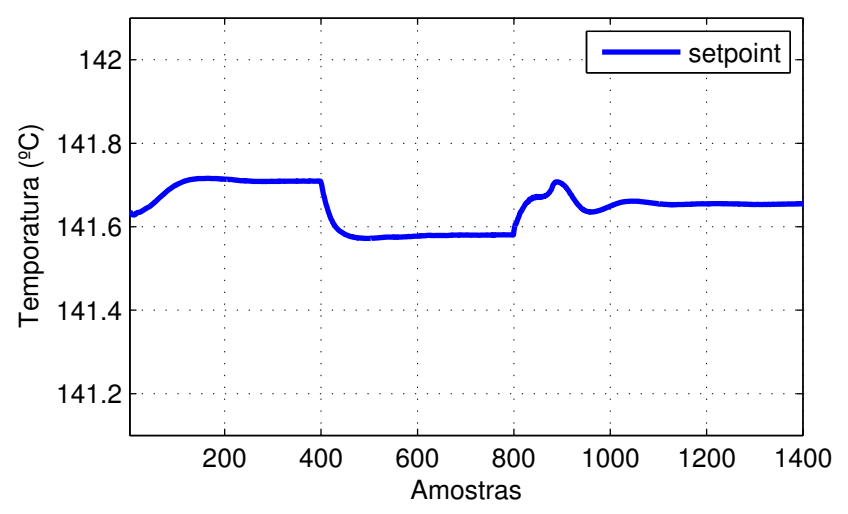

Figura 9. Set Point gerado pelo sistema fuzzy para o controlador TIC-102

Pode-se observar que os sinais de controle gerados permaneceram suaves e constantes durante maior parte do tempo de simulação, com alguns pequenos sobre picos durante o período de transição das referências.

Alguns parâmetros numéricos de desempenho são apresentados na Tabela 7. São considerados piores casos os in- 
tervalos de simulação onde observou-se os maiores valores para os índices descritos.

Tabela 7. índices de desempenho (Piores Casos)

\begin{tabular}{ccc} 
índice & valor & Intervalo \\
\hline Overshoot & $25 \%$ & $0-400$ \\
Tempo de subida & $2310 \mathrm{~s}$ & $800-1400$ \\
Tempo de Acomodação & $9000 \mathrm{~s}$ & $800-1400$ \\
Erro de regime & $1.9 \times 10^{-5}$ & $800-1400$ \\
\hline
\end{tabular}

Como pode ser observado através dos dados e gráficos apresentados, o sistema ajustou a quantidade de i-pentano para todos os valores de referência com um erro de regime permanente baixo, de aproximadamente $1.9 \times 10^{-5}$.

\section{CONCLUSÃO}

O controlador baseado em lógica fuzzy é uma técnica eficiente na realização de controle avançado. Porém, a necessidade da realização de medições a cada período de amostragem de 30 segundos seria inviável através de um processo de cromatografia tradicional. Propôs-se então, um sensor virtual para estimar as frações molares de i-pentano retirando a necessidade de se realizar uma cromatografia a todo período de amostragem.

Portanto, sistemas de inferência baseado em rede neural artificial é uma poderosa ferramente de medição de variáveis difíceis de serem mensuradas, viabilizando a realização de um controle em tempo real de elementos contaminantes no Gás Liquefeito de Petróleo.

Os resultados demonstram que o sistema sistema de inferência utilizando redes neurais artificiais viabilizou o controle avançado do i-pentano. O controlador fuzzy, por sua vez, ajustou a fração molar para o valor desejado com um erro de regime muito próximo de zero. Conclui-se então, que o controle realizado foi satisfatório e os resultados pretendidos foram obtidos.

\section{REFERÊNCIAS}

Campos, M.C., Gomes, M.V., Moreira, V.D., Lima, M.F., and Silva, J.R. (2009). Advanced control and optimization of a natural gas plant-benefits of the new regulatory control strategy. In Computer Aided Chemical Engineering, volume 27, 1683-1688. Elsevier.

El-Nagar, A.M. (2018). Nonlinear dynamic systems identification using recurrent interval type-2 TSK fuzzy neural network - A novel structure. ISA Transactions, 72, 205217. doi:10.1016/j.isatra.2017.10.012.

Fortuna, L., Graziani, S., and Xibilia, M.G. (2005). Soft sensors for product quality monitoring in debutanizer distillation columns. doi:10.1016/j.conengprac.2004.04. 013.

Haykin, S. (2001). Redes Neurais: Princípios e Prática. Bookman, Porto Alegre/RS - Brasil, 2 edition.

Juang, C.F., Jhan, Y.H., Chen, Y.M., and Hsu, C.M. (2017). Evolutionary wall-following hexapod robot using advanced multi-objective continuous ant colony optimized fuzzy controller. IEEE Transactions on Cognitive and Developmental Systems.
Junior, J.M.d.A., Linhares, L.L.d.S., and Araújo, F.M. (2007). Artificial intelligence techniques applied in a simulated oil distillation system. In COBEM-19th International Congress of Mechanical Engineering.

Klir, G.J. and Folger, T.A. (1988). Fuzzy sets, uncertainty, and information.

Mendes, W.R., Araújo, F.M.U., Dutta, R., and Heeren, D.M. (2019). Fuzzy control system for variable rate irrigation using remote sensing. Expert Systems with Applications, 124, 13-24.

Norgaard, M., Ravn, O., Poulsen, N.K., and Hansen, L.K. (2001). Neural Network for modelling and control of dynamic systems. Springer-Verlag London Limited, Londes - Inglaterra.

Pasieka, M., Grzesik, N., and Kuźma, K. (2017). Simulation modeling of fuzzy logic controller for aircraft engines. International Journal of Computing, 16(1), 2733.

Rani, A., Singh, V., and Gupta, J.R.P. (2013). Development of soft sensor for neural network based control of distillation column. ISA Transactions, 52(3), 438-449. doi:10.1016/j.isatra.2012.12.009.

Sousa, A.L., Pires, A.H., and Araújo, F.M. (2017). Controlador fuzzy multivariável aplicado ao processo de produção do gás liquefeito de petróleo. XIII Simpósio Brasileiro de Automação Inteligente.

Takagi, T. and Sugeno, M. (1993). Fuzzy identification of systems and its applications to modeling and control. In Readings in Fuzzy Sets for Intelligent Systems, 387-403. Elsevier.

Tavoosi, J., Suratgar, A.A., and Menhaj, M.B. (2016). Nonlinear system identification based on a selforganizing type-2 fuzzy RBFN. Engineering Applications of Artificial Intelligence, 54, 26-38. doi:10.1016/j. engappai.2016.04.006.

Vijaya Raghavan, S.R., Radhakrishnan, T.K., and Srinivasan, K. (2011). Soft sensor based composition estimation and controller design for an ideal reactive distillation column. ISA Transactions, 50(1), 61-70. doi:10.1016/j. isatra.2010.09.001.

Zadeh, L.A. (1965). Fuzzy sets. Information and control, $8(3), 338-353$. 